\section{CONSOLIDACIÓN DE LAS METODOLOGÍAS ACTIVAS EN EDUCACIÓN FÍSICA EN LAS ESCUELAS DE ENSEÑANZA SECUNDARIA}

\author{
CONSOLIDAÇÃO DAS METODOLOGIAS ATIVAS EM EDUCAÇÃO FÍSICA NAS \\ ESCOLAS DE ENSINO MÉDIO
}

\author{
CONSOLIDATION OF ACTIVE METHODOLOGIES IN PHYSICAL EDUCATION AT \\ SECONDARY SCHOOL
}

\author{
Jorge Agustín Zapatero*, María Dolores González Rivera**, \\ Antonio Campos Izquierdo***
}

\begin{abstract}
Resumen: El sistema educativo español se sumó a un movimiento de reforma educativa, donde las competencias a adquirir pretenden promover el uso de estilos de enseñanza que impulsen la autonomía del alumno, la interacción entre alumnos y su participación activa en los procesos de enseñanza y aprendizaje. El objetivo de este estudio es analizar la realidad de estos estilos de enseñanza activos, conociendo en profundidad los modelos de enseñanza que se aplican en la Educación Física. Participaron un grupo de profesores de secundaria de la Comunidad de Madrid. Se empleó metodología cualitativa. Se recurrió a la técnica de análisis de contenido, discusión en grupo, observación y entrevistas, triangulando los datos recogidos con Atlas.ti. Los resultados sugieren que, a pesar de que los docentes reconocen los beneficios de la enseñanza activa, donde se proporciona autonomía al estudiante, en sus prácticas profesionales predominan estilos de enseñanza instructiva, a través de modelos reproductivos.
\end{abstract}

\section{Palavras chave:} Educação baseada em competências.

Ensino.

Docentes.

Ensino fundamental e médio.
Resumo: O sistema educativo espanhol juntou-se a um movimento de reforma educacional, em que as competências a adquirir pretendem promover a utilização dos estilos de ensino, que promovem a autonomia dos alunos, a interação entre esses e sua participação ativa nos processos de ensino. 0 objetivo deste estudo foi analisar a realidade desses estilos de ensino ativo, sabendo em profundidade quais os modelos de ensino aplicados em Educação Física. Os participantes pertenciam a um grupo de professores do ensino médio da Comunidade de Madrid. Foi utilizada uma metodologia qualitativa. Recorreu-se à técnica de análise de conteúdo, discussão em grupo, observação e entrevistas, tendo estes meios de recolha de informação sido triangulados, utilizando-se o Atlas.ti. Os resultados sugerem que, embora os professores tenham conhecimento dos benefícios dos estilos de ensino ativo, no qual é proporcionada a autonomia ao estudante, nas suas práticas profissionais predominam estilos de ensino instrutivo, através de modelos reprodutivos.

\footnotetext{
Abstract: Spain's educational system joined an educational reform movement based on competencies, which promotes teaching styles that encourage student's autonomy, interaction and active participation in teaching and learning processes. This study looks into the reality of these active teaching styles and intends to know the methodology applied in Physical Education in depth. Participants were a group of secondary school teachers from the Community of Madrid. Qualitative methodology was employed, as well as content analysis, focus group, observation and interviews, while triangulating data using Atlas.ti. The findings suggest that while teachers acknowledge the benefits of active teaching that provides students with autonomy, traditional, reproductive and directing teaching styles prevail in their professional practices.
}

*Universidad Complutense de Madrid. Madrid, España. E-mail: jzapater@ucm.es

**Universidad de Alcalá. Alcalá de Henares, España. E-mail: marilin.gonzalez@uah.es

***Universidad Politécnica de Madrid. Madrid, España.

E-mail: antonio.campos.izquierdo@ upm.es

Recebido em: 24-12-2016 Aprovado em: 30-04-2018

DOI: http://dx.doi.org/10.22456/1982-8918.70291 (c) (1) (8) Licence 


\section{INTRODUCCIÓN}

Las competencias aparecieron en el discurso educativo internacional en la última década del siglo XX, con los objetivos de acercar la formación al mercado laboral y desarrollar la capacidad de "aprender a aprender" en una sociedad que se consideró "la sociedad de la información" (JALLADE, 2011; KLEIBRINK, 2011). En Latinoamérica las competencias fueron debatidas en el marco de políticas educativas neoliberales (CONFEDERAÇÃO NACIONAL DOS TRABALHADORES EM EDUCAÇÃO, 2005). En este contexto, la llegada de las competencias al discurso educativo no estuvo exenta de críticas, entre otras razones, por su cercanía al mercado laboral, su carácter globalizador y, en otras ocasiones, por el bajo ejercicio crítico con el que se incorporó a los currículos nacionales (FIGUERASet al., 2016; MARTINS et al., 2013). En este caso, los expertos gubernamentales de cada país adaptaron su normativa con orientaciones distintas en lo que respecta a las competencias (MARTINS et al., 2013). Concretamente en España y en niveles preuniversitarios, la Ley Orgánica 2/2006, de 3 de mayo, de Educación modificada por la Ley Orgánica 8/2013, de 9 de diciembre, para la mejora de la calidad educativa (LOE-LOMCE) potenció la necesidad de contribuir a las competencias clave desde todas las asignaturas y, entre ellas, la Educación Física (EF).

No obstante, más allá del debate en la incorporación de las competencias a la normativa y los programas educativos, este enfoque tuvo una repercusión sobre el "currículo en acción" y la forma de desempeñar del profesor de EF (MONARCA; RAPPOPORT, 2013). En España, el modelo impulsó algunos cambios en ámbitos como la organización escolar, las planificaciones educativas, la evaluación y la metodología de enseñanza. A nivel organizativo, las competencias son elementos comunes a todas las asignaturas, por lo que los planteamientos en los centros educativos deben ser interdisciplinares, garantizando la coordinación y el trabajo entre asignaturas (MONARCA; RAPPOPORT, 2013). En relación con la planificación educativa, las competencias deben servir de referencia para diseñar programas educativos coherentes en las escuelas (HORTIGÜELA; ABELLA; PÉREZ-PUEYO, 2015a). Los contenidos educativos son imprescindibles, pero no tienen un valor en sí mismos, sino en la medida en que sirvan para resolver problemas cercanos a la vida cotidiana del alumno. En cuanto a la evaluación, las competencias exigen la identificación de comportamientos observables, que favorezcan una evaluación objetiva y, además, requieren de una orientación formativa, que incluya al alumno en su propia evaluación (ZAPATERO-AYUSO; GONZÁLEZ-RIVERA; CAMPOSIZQUIERDO, 2012). Por último, el ámbito metodológico se convirtió en una de las piedras angulares para el desarrollo de este modelo educativo, siendo una preocupación relevante para su implementación (ION; CANO, 2012; MÉNDEZ-GIMÉNEZ; SIERRA; MAÑANA, 2013).

El cambio metodológico fue exigido a todas las asignaturas y entre ellas a la EF. De este modo, el profesor de EF debía replantearse qué enseñar en la asignatura y, de forma no menos relevante, debía cuestionarse cómo enseñar esos contenidos. La metodología competencial favorece que el alumno gestione y aplique el conocimiento en situaciones reales y cambiantes, así como sitúa al alumno en el centro del proceso de enseñanza, otorgándole autonomía y responsabilidad (TEJADA, 2012). Este hecho deriva en un giro en la función docente, siendo el profesor un "facilitador de conocimiento", que acompaña y orienta al alumno (ION; CANO, 2012). Además, la metodología competencial ofrece posibilidades para que el alumno interaccione y tome decisiones a través de métodos como el aprendizaje cooperativo (FERNÁNDEZ-RIVAS; ESPADA, 2016). 
No obstante, a pesar de que este cambio metodológico apareció ligado a la reforma competencial, la EF se viene preocupando por su metodología y los estilos de enseñanza desde hace casi medio siglo y en la actualidad sigue siendo un problema de alcance internacional (GOLDBERGER; ANSWORTH; BYRA, 2012). De hecho, el modo de enseñar en EF parece una cuestión de carácter epistemológica, cuya evolución va ligada a la identidad de esta materia, la cual posee un largo recorrido desde un comienzo de carácter técnico y reproductivo hasta las actuales perspectivas más transformacionales, deliberativas o críticas (OLIVEIRA, 1997; SOARES, 1992).Concretamente en España, siguiendo las ideas de Mosston y Answorth (1990), la indagación sobre la forma de enseñar en EF proliferó a partir de la aparición de los trabajos de Delgado-Noguera y Sicilia-Camacho (SICILIA-CAMACHO, 2001; SICILIA-CAMACHO; DELGADO-NOGUERA, 2002). Siguiendo a estos autores, la "teoría de los estilos de enseñanza "organizó la relación profesor y alumno en un "espectro de estilos", desde aquellos de máxima directividad del docente y mínima decisión del alumno (estilos de enseñanza tradicionales) hasta aquellos en los que el alumno es el máximo protagonista (estilos creativos).Entre ambos polos, se encuentran los estilos que fomentan la individualización, participación y socialización del alumno y que, por su carácter activo, mantienen una relación directa con el enfoque por competencias.

En el marco normativo competencial, la adopción de estos estilos de enseñanza (activos, participativos e individualizadores) constituye una prescripción normativa en España. Ahora bien, la inclusión de estos estilos se justifica también en las ventajas que reportan al alumnado. Laal, Khattami-Kermanshahi y Laal (2014) encontraron como el aprendizaje colaborativo proporcionaba importantes beneficios sociales, psicológicos y académicos. En la misma línea, Corina, Neacsu, Georgiana y Suditu (2011) obtuvieron que la enseñanza en entornos activos favorecía el aprendizaje y la creatividad de los alumnos. Los hallazgos de Álvarez-Arregui, Rodríguez-Martín y San-Fabián (2013) y Amin y Rajaei (2011) informaron que los alumnos prefieren estilos activos, lo que adquiere una mayor relevancia si la satisfacción por el estilo parece ligada a un rendimiento mayor (CORINA et al., 2011). Además, los profesores de EF tienen una actitud positiva hacia estos estilos cooperativos y planteamientos competenciales (AMIN; RAJAEI, 2011; FERNÁNDEZ-RIVAS; ESPADA, 2016; HORTIGÜELA et al., 2015a, 2015b; MÉNDEZ-GIMÉNEZ et al., 2013)

Sin embargo, en España los estudios vienen evidenciando que esta reforma competencial no llega a la práctica (HORTIGÜELA et al., 2015a, 2015b; MÉNDEZ-ALONSO; MÉNDEZ-GIMÉNEZ; FERNÁNDEZ-RÍO, 2015; MONARCA; RAPPOPORT, 2013). En la misma línea, en contraposición con la amplia preocupación sobre los estilos de enseñanza en EF, las investigaciones constatan la baja materialización de los estilos activos, participativos y centrados en el alumno en el desempeño diario de los docentes de EF (AKTOP; KARAHAN, 2012; ARAUJO; CAMPOS, 2006; COTHRAN et al., 2005; HEIN et al., 2012; HODGES; COTHRAN, 2003). Diversos estudios profundizaron sobre las causas que dificultan la incorporación de esta metodología más activa. Al respecto, la EF se encuentra con limitantes formativos y organizativos y escasos apoyos en la implementación de políticas educativas (FERREIRA; PEREIRA, 2009; MÉNDEZ-GIMÉNEZ et al., 2013; RAMÍREZ, 2015; VARGAS;SILVA, 2008; ZAPATERO-AYUSO et al., 2012).

De acuerdo con estos resultados, este estudio pretende conocer en profundidad el modo en que se concreta y desarrolla la metodología en los departamentos de EF de un grupo de profesores de secundaria de la Comunidad de Madrid(España). Se persiguen los siguientes objetivos específicos: 
a) indagar sobre las creencias y actitudes del profesorado participante acerca de las metodologías de enseñanza competencial, más participativas y activas.

b) analizar la metodología desarrollada en el quehacer diario de los profesores de EF.

c) conocer el grado de relación entre las creencias de los participantes sobre estas metodologías y su desarrollo en las aulas.

\section{METODOLOGÍA}

La metodología de investigación se encuadra en el paradigma cualitativo para acercarse a una realidad educativa compleja, evolutiva, dinámica, intangible, vivenciada e interactiva (COLÁS; BUENDÍA, 1992; PÉREZ-SERRANO, 2004). Para asegurar el rigor del estudio se siguieron criterios de credibilidad, transferibilidad, dependencia y confirmabilidad. La credibilidad se vincula con el isomorfismo entre los datos obtenidos y la realidad. La aplicabilidad se relaciona con la transferibilidad del procedimiento de investigación en situaciones idénticas. La dependencia es entendida como la capacidad para replicar el estudio y obtener los mismos hallazgos en los mismos contextos. La confirmabilidad se vincula con la neutralidad de los datos obtenidos, sin estar influenciados por el investigador (BISQUERRA, 1989; COLÁS; BUENDÍA, 1992).

Deacuerdocon estos criteriosse desarrollaron diversas actuaciones enlaimplementación de la investigación. Se analizaron documentos oficiales internos (las programaciones de los departamentos) y se contrastaron con la aplicación de los mismos a través de una observación prolongada y persistente. Se utilizaron categorías estables para analizar la metodología de enseñanza, las cuales estaban basadas en una revisión normativa y bibliográfica sobre los estilos de enseñanza en EF. Además se aplicó la triangulación con el objetivo de recoger y analizar la información desde distintas perspectivas para contrastar e interpretar los datos, dotando al estudio del conveniente rigor y credibilidad (COLÁS; BUENDÍA, 1992).Siguiendo a Pérez-Serrano (2004), la triangulación fue de técnicas, de fuentes, de espacios y de investigador. En concreto, esta investigación trianguló las técnicas de análisis de contenido, observación, grupo de discusión y entrevistas semiestructuradas. Estas técnicas recogían información de diferentes fuentes (programas educativos, profesores y observador externo) y en diferentes espacios (cuatro centros educativos de diferentes zonas de la Comunidad de Madrid). El diseño y aplicación de los instrumentos de investigación fue contrastado y revisado constantemente entre los investigadores.

\subsection{Participantes}

Para la selección de los participantes se siguieron criterios con el fin de conseguir una heterogeneidad y, al mismo tiempo, respetar cierta homogeneidad (CALLEJO, 2001; KRUEGER, 1999). Se establecieron criterios como:
a) participación de ambos sexos.
b) diferentes años de experiencia docente.
c) dispersión geográfica en la Comunidad de Madrid.
d) disponibilidad para participar en el estudio.

En cuanto al procedimiento, se visitaron los centros educativos por contactos y fueron ampliados por medio de la técnica de bola de nieve (MARTíNEZ-SALGADO, 2012; 
RUIZ-OLABUÉNAGA, 1999). Una vez allí, se contactó con el profesorado de EF y, cuando se confirmó que reunían los criterios de selección, se les invitó a participar en el estudio. Conforme a cuestiones éticas, se les informó del proceso de investigación, sus objetivos y del carácter anónimo y confidencial de los hallazgos. Se comunicó esta información a sus equipos directivos y se obtuvo su correspondiente autorización para desarrollar la investigación.

De este modo se consiguió la participación de seis docentes de la Comunidad de Madrid en Educación Secundaria Obligatoria (ESO). En España esta etapa educativa abarca desde los 12 a los 16 años y se estructura en cuatro niveles educativos ( $1^{\circ}$ a $4^{\circ}$ ESO). En sus clases participaron sus respectivos alumnos $(n=723$ ). Los docentes 1 y 2 y los docentes 3 y 4 compartían centro educativo y Departamento (centro educativo 1 y 2 , respectivamente). Los docentes 5 y 6 no compartían centro educativo (centro educativo 3 y 4 , respectivamente). De acuerdo con los criterios de selección, las características de los profesores fueron:

a) sexo: cuatro hombres y dos mujeres.

b) experiencia profesional: dos de ellos noveles (menos de dos años de experiencia) y cuatro expertos (más de 15 años).

c) dispersión geográfica: centros educativos de Madrid-Capital (centro), Madrid-Sur, Madrid-Norte y Madrid-Este.

d) todos mostraron una adecuada predisposición para participar en el estudio.

\subsection{Instrumentos y procedimiento}

Se aplicaron las técnicas de análisis de contenido, observación, grupo de discusión y entrevistas para analizar los objetivos de la investigación. Se llevaron a cabo en el último tramo del curso académico para garantizar que profesores y alumnos estuvieran familiarizados. El cuadro 1 resume este proceso de forma estructurada. Tras este cuadro, se expondrá el procedimiento de desarrollo de las técnicas de investigación.

Cuadro 1 - Resumen del proceso de investigación

\begin{tabular}{|c|c|c|}
\hline Objetivos de la investigación & Técnicas & Fuentes \\
\hline $\begin{array}{c}\text { a) indagar sobre las creencias y actitudes } \\
\text { del profesorado participante acerca de las } \\
\text { metodologías de enseñanza competencial, más } \\
\text { participativas y activas }\end{array}$ & Análisis de contenido & $\begin{array}{c}\text { Grupo de discusión } \\
\text { Entrevistas } \\
\text { Departamento }\end{array}$ \\
\cline { 2 - 3 } $\begin{array}{c}\text { b) analizar la metodología desarrollada en el } \\
\text { quehacer diario de los profesores de EF }\end{array}$ & Observación externa & Participantes \\
\hline $\begin{array}{c}\text { c) conocer el grado de relación entre las creencias } \\
\text { sobre estas metodologías y su desarrollo en las } \\
\text { aulas de los participantes }\end{array}$ & $\begin{array}{c}\text { Interpretación justificada } \\
\text { en los resultados de los } \\
\text { objetivos a) y b) }\end{array}$ & Investigador \\
\hline
\end{tabular}

Fuente: Elaboración propia

El análisis de contenido se aplicó en primer lugar con el objetivo de familiarizar al investigador con el programa educativo que se observaría con posterioridad (ALVIRA, 1991). La normativa educativa española prescribe la necesidad de que los departamentos, entre ellos el de EF, recojan su planteamiento educativo en un documento: la programación didáctica. Esta programación debe respetar la jerarquía curricular y realizarse de acuerdo a las ideas del centro educativo y la legislación. Por tanto, supone una expresión de las ideas y creencias del 
profesorado. Con esta justificación y para responder al primer objetivo de estudio, se desarrolló un análisis de contenido de las programaciones de Departamento de los participantes. Concretamente el procedimiento de análisis de contenido siguió las orientaciones de Bardin (1986), Krippendorff (1990) y Mendonça, Soares y Eliete (2010) para la configuración del corpus documental, el preanálisis y el análisis e interpretación posterior. En primer lugar, se configuró el corpus documental (las cuatro programaciones didácticas de los participantes, cuadro 2) y se llevó a cabo una lectura exploratoria. Posteriormente, se determinó la unidad de análisis (las programaciones preparadas en formato digital), de registro (frase contenida entre dos puntos, que se entendió circunscrita a la unidad de contexto, el párrafo) y las reglas de enumeración (presencia o ausencia de un código y su frecuencia de aparición).

Cuadro 2 - Corpus documental del análisis de contenido y su vinculación con los participantes y sus contextos

\begin{tabular}{|c|c|c|c|}
\hline Programación didáctica & $\begin{array}{c}\text { Departamento de Educación } \\
\text { Física }\end{array}$ & Centro Educativo & Docentes \\
\hline Programación 1 & Departamento 1 & Centro 1 & Docente 1 \\
\cline { 4 - 4 } & Departamento 2 & Centro 2 & Docente 2 \\
\cline { 3 - 4 } Programación 2 & Departamento 3 & Docente 4 \\
\hline Programación 3 & Departamento 4 & Centro 3 & Docente 5 \\
\hline Programación 4 & Centro 4 & Docente 6 \\
\hline
\end{tabular}

Fuente: Elaboración propia

La observación fue la actividad principal para valorar las metodologías empleadas en el desarrollo de los programas. Se registraron un total de 190 sesiones entre los seis participantes (Docente 1=34; Docente 2=41; Docente 3=32; Docente 4=33; Docente 5=23 y Docente 6=27). Previamente se llevó a cabo una prueba piloto de la observación para reducir el sesgo de reactividad, formar al observador y optimizar el proceso de recogida de información (ARNAU; ANGUERA; GÓMEZ, 1990).La técnica de observación fue no participante y se registró a través de notas de campo (ARNAU etal., 1990). Este instrumento fue construido ad hoc siguiendo las orientaciones de Patton (1987).Las notas de campo recogían información acerca del contexto (docente observado, nivel educativo, fecha, lugar, etc.) y las actividades de enseñanza que se desarrollaban en la sesión; así como incorporaban un registro categorial sobre los estilos de enseñanza.

El grupo de discusión se aplicó previamente a las entrevistas, pues puede servir como base para el desarrollo de otras técnicas (GIL-FLORES, 1993). El procedimiento atendió a las orientaciones de Callejo (2001), Ibáñez (2003) y Krueger (1991). Los criterios de selección de los participantes garantizaron cierta heterogeneidad dentro de la homogeneidad requerida para la fluidez del discurso. El número de participantes, seis, respondía a las sugerencias de estos autores. Se llevó a cabo una primera sesión inicial de presentación, exposición de propósitos y normas básicas y recogida de consentimientos informados con sus respectivas firmas. Posteriormente, la fase de desarrollo se estructuró en dos sesiones de 70 y 80 minutos. Estas se desarrollaron en un aula libre de ruidos e interferencias en la Universidad Politécnica de Madrid y fueron grabadas en audio.

Las entrevistas semiestructuradas se aplicaron en último lugar, pues son útiles para complementar la información recogida con otras técnicas (LUKAS; SANTIAGO, 2004; MARTÍNEZ-MEDIANO, 2004). Su objetivo fue profundizar sobre el primer objetivo de estudio, que ya fue indagado a través del análisis de contenido y el grupo de discusión. Se siguieron los 
procedimientos de Arnau et al. (1990), Lukas y Santiago (2004) y Valles (2002). Se obtuvieron seis entrevistas semiestructuradas (una con cada participante) siguiendo un guion previamente establecido. El lugar y el momento fueron elegidos en función de los requerimientos de los informantes, en un lugar confortable y donde el nivel de ruido no afectara a la legibilidad de la información recabada. Tuvieron lugar en sus centros educativos y la duración de las entrevistas osciló entre 30 minutos y 1 hora. Estas fueron registradas en audio.

Con respecto al grupo de discusión y las entrevistas, para atender a los objetivos de este estudio, las cuestiones de investigación se estructuraron en guiones tentativos y abiertos. Estos siguieron una lógica de embudo, fueron diseñado a partir de una revisión normativa y de expertos (referencias sobre el problema de estudio)y se validaron mediante una verificación intersubjetiva (triangulación de investigador) o revisión entre los investigadores participantes en el estudio (PÉREZ-SERRANO, 1994).

\subsection{Análisis y tratamiento de la información}

Los datos recogidos fueron tratados cualitativamente usando el software Atlas.ti. Se emplearon procedimientos inductivos, para el análisis de contenido, grupo de discusión y entrevistas, y deductivos, para las notas de campo.

La codificación del análisis de contenido, el grupo de discusión y las entrevistas siguió algunos de los procedimientos del modelo "Grounded Theory" (Teoría Fundamentada) de Glaser y Strauss (1967), en el que se fundamenta Atlas.ti. Se aplicaron tres codificaciones sucesivas (abierta, axial y selectiva) hasta alcanzar la saturación teórica (BARRACHINA; BLASCO, 2012; STRAUSS; CORBIN, 2002). Los códigos obtenidos se presentan en los resultados (figuras 1 y 2), utilizando la herramienta "Network" de Atlas.ti.

La codificación de las notas de campo fue deductiva y se basó en las categorías o códigos preestablecidos en este instrumento (cuadro 3). La elección de estas categorías fue justificada en los trabajos de Sicilia-Camacho y Delgado-Noguera acerca de los estilos de enseñanza en EF (SICLIA-CAMACHO, 2001; SICILIA-CAMACHO; DELGADO-NOGUERA, 2002). Se asignó una cita al respectivo código siempre que el investigador observara el desarrollo de alguno de los estilos de enseñanza en la sesión. Para la presentación de los resultados, se llevó a cabo una computación de frecuencias y porcentajes, exportando los resultados de Atlas.ti a Excel.

Cuadro 3 - Códigos sobre estilos de enseñanza en la EF

\begin{tabular}{|c|c|c|c|c|}
\hline Tradicionales & Individualizadores & $\begin{array}{c}\text { Participativos y } \\
\text { socializadores }\end{array}$ & Cognoscitivos & Creativos \\
\hline $\begin{array}{c}\text { Mando directo } \\
\text { Mando directo } \\
\text { modificado } \\
\text { Asignación de tareas }\end{array}$ & $\begin{array}{c}\text { Programas individuales } \\
\text { Enseñanza programada } \\
\text { Trabajo por grupos de nivel } \\
\text { o interés } \\
\text { Enseñanza modular }\end{array}$ & $\begin{array}{c}\text { Enseñanza recíproca } \\
\text { Microenseñanza } \\
\text { Grupos reducidos } \\
\text { Dinámicas de grupo }\end{array}$ & $\begin{array}{c}\text { Resolución de } \\
\text { problemas } \\
\text { Descubrimiento } \\
\text { guiado }\end{array}$ & Sinéctica \\
\hline
\end{tabular}

Fuente: Sicilia-Camacho (2001); Sicilia-Camacho; Delgado Noguera (2002)

\section{RESULTADOS}

A continuación se presentarán los resultados en función de los dos primeros objetivos de estudio, dado que el tercer objetivo se vinculó con el tratamiento de los dos primeros. Este tercer objetivo se abordará en la discusión de los resultados. 


\subsection{Concepciones y creencia del profesorado sobre la metodología de enseñanza por competencias}

las concepciones y creencias del profesorado fueron exploradas mediante el análisis de contenido de las programaciones, el grupo de discusión y las entrevistas. A continuaciónse presentan los resultados del análisis de contenido (en primer lugar) y de las entrevistas y el grupo de discusión (en segundo lugar).

El análisis de las programaciones de los Departamentos obtuvo el conjunto de códigos y relaciones que forman parte de la "Teoría Fundamentada" (figura 1), cuyos aspectos más relevantes se desarrollan tras la misma.

Figura 1 - Principios metodológicos planificados en las programaciones de los participantes a través del análisis de contenido

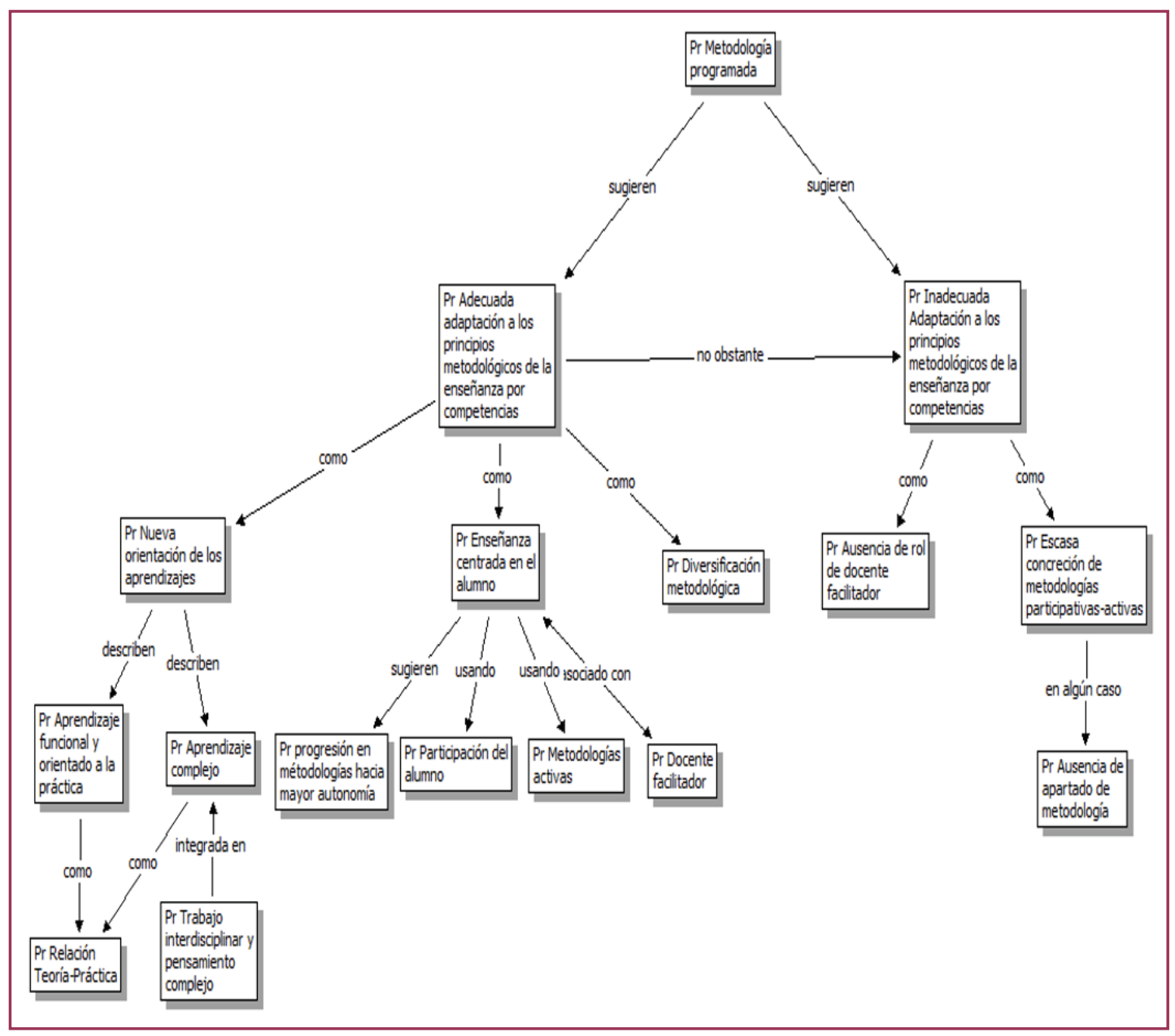

Fuente: Elaboración propia

Los resultados sugirieron, mayoritariamente, una adecuada adaptación a los principios metodológicos de la enseñanza por competencias. En esta línea, en sus programaciones, los participantes recogieron ideas sobre la orientación compleja, holística, interdisciplinar y funcional de los aprendizajes:

Habrá que tener en cuenta las posibles transferencias de unos contenidos a otros así como la interrelación de nuestra área con las otras del currículo con el fin de no presentar el conocimiento en departamentos estancos sino globalmente. 
Se pretende facilitar al alumno/a una visión integradora de la asignatura a través de actividades y enfoques multidisciplinares, tomando siempre como punto de partida la realidad del individuo.(Programa 2, análisis de contenido).

Además, las programaciones abogaron por una metodología centrada en el alumno, en la cual se otorga protagonismo al mismo y el profesor se convierte en un facilitador. Las siguientes citas son ejemplos de estos resultados:

El profesor debe considerarse como un intermediario entre el alumno/a y el nuevo conocimiento. (Programa 1, análisis de contenido).

La EF requiere una metodología fundamentalmente activa, no solo implicando el activismo motor, sino haciendo que el proceso enseñanza-aprendizaje fomente la reflexión y el análisis del alumno/a acerca de los objetivos planteados y le dé la oportunidad de ejercitar su responsabilidad mediante la toma de decisiones. (Programa 2, análisis de contenido).

Esto se verá facilitado por el empleo de estilos de enseñanza caracterizados por la implicación de los alumnos en el proceso de enseñanza-aprendizaje como la enseñanza recíproca, grupos reducidos y/o la microenseñanza.(Programa 3 , análisis de contenido).

En cuanto a la opinión expuesta en las entrevistas y el grupo de discusión, el análisis permitió la identificación de los códigos y relaciones expuestos en la figura 2. A continuación se analizan algunos de sus aspectos más relevantes.

Figura 2 - Concepciones y creencias sobre las metodologías de enseñanza por competencias a través del grupo de discusión y las entrevistas

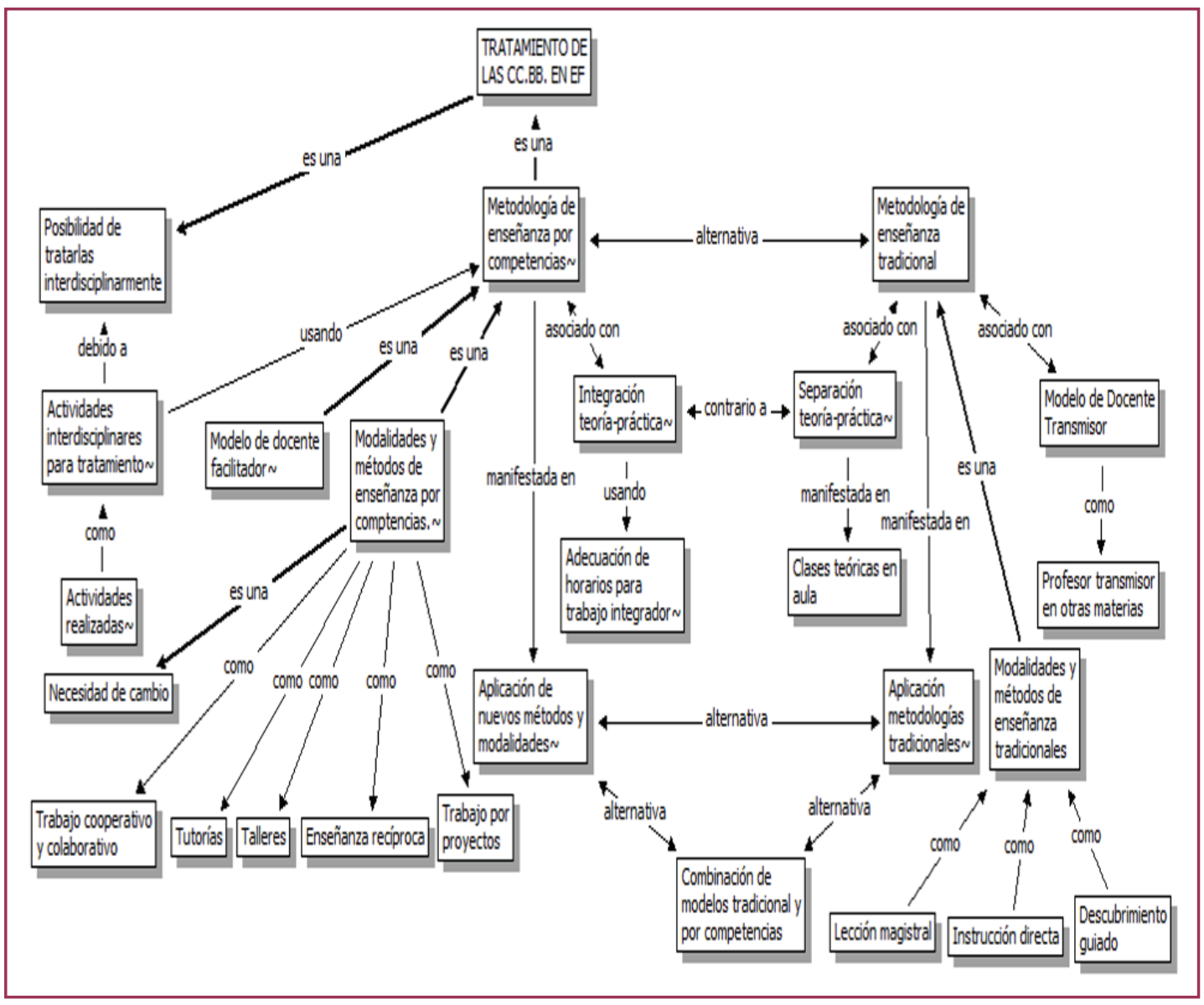

Fuente: Elaboración propia 
Los participantes sugirieron la necesidad de cambiar la metodología hacia postulados de la enseñanza por competencias:

Yo creo que para llevar a cabo este tipo de cosas, las metodologías a utilizar deben ser diferentes a las de toda la vida, que el mando directo [...] (Docente 4, entrevista).

[...] trabajar en estas competencias es diluir la importancia que tienen los contenidos, darle más importancia a estas capacidades y al trabajar esas capacidades lo que tienes que hacer es un cambio metodológico. El profesor ya no es el protagonista de las clases [...] (Docente 5, entrevista).

Asimismo, los profesores conocían aspectos que tienen que ver con el modelo, como el diseño de actividades interdisciplinares, el trabajo cooperativo o por proyectos, afirmando desarrollar algunos de estos planteamientos. La siguiente cita desarrolla un ejemplo de este tratamiento interdisciplinar:

Actividades extraescolares, excursiones que programen desde los distintos departamentos; por ejemplo, [...]la salida a hacer senderismo y vas con el de historia y el de geología y ahí intentas un poco hacer algo de interdisciplinariedad. (Docente 5, grupo de discusión).

Además, los participantes afirmaron que la formación de los profesores de EF puede facilitar el desarrollo de estas metodologías:

En EF nos parece normal como trabajamos nosotros, pero no es lo normal como trabajamos nosotros. Porque nosotros tenemos una formación en ese sentido bastante completa. (Docente 2, entrevista).

Yo insisto en que los de EF no; pero, la mayoría de los profesores [...] se han visto agobiados por este paso de ser transmisor de conocimientos [...] porque se creen que son transmisores de conocimientos y fuera. (Docente 4, grupo de discusión).

No obstante, en este estudio aparecieron opiniones que apuntaron a que este tipo de metodologías no se desarrollaban de manera frecuente: Otros no saben menos que nosotros, [...] pero, a la hora de la verdad muchos aplicamos las mismas metodologías que todos los demás [...] (Docente 4, entrevista).

De forma complementaria a este planteamiento, otros participantes sugirieron combinar el uso de ambos modelos, el más tradicional, centrado en el contenido y el docente y, el competencial, más activo y centrado en el alumno:

Yo creo que también el profesor tiene que tener un conocimiento y poder transmitirlo. Y no creo que sea excluyente tampoco. [...] No creo que la lección magistral quede excluida y que la pizarra quede excluida, y que ya solo las nuevas tecnologías sirvan. No. En muchas ocasiones puede seguir recurriendo a lo otro y es tan válido como, a lo mejor, el mejor programa informático. (Docente 2, entrevista).

Si estamos hablando de autonomía, tú no puedes dar todas las sesiones teledirigidas. ¡Ojo! Lo que no quiere decir que no te valgas de la instrucción directa en muchas ocasiones. (Docente 1, entrevista).

\subsection{La metodología desarrollada por los participantes}

La observación de las sesiones de los participantes permitió conocer los estilos de enseñanza utilizadas por los mismos (tabla1). En lo que respecta a estos estilos de enseñanza, 
los tradicionales fueron los más aplicados en las sesiones observadas $(69,72 \%$ de las citas). De forma notablemente reducida, los participantes utilizaron estilos individualizadores (18,81\% de las citas) y creativos (7,34\% de las citas). Los estilos de enseñanza participativos y socializadores $(3,67 \%$ de las citas) y los cognoscitivos $(0,46 \%$ de las citas) fueron los menos empleados (tabla 1).

Tabla 1 - Estilos de enseñanza utilizados por los participantes (en porcentaje de citas)

\begin{tabular}{llcc}
\hline Tipos de estilos de enseñanza (\% de citas) & Estilos de enseñanza & $\begin{array}{c}\% \text { de } \\
\text { citas }\end{array}$ & Total \\
\hline Tradicionales & Asignación de tareas & $90,13 \%$ & \\
$(69,72 \%)$ & Mando directo modificado & $7,24 \%$ & $100 \%$ \\
& Mando directo & $2,63 \%$ & \\
& Trabajo por grupos de nivel o & $95,12 \%$ & \\
Individualizadores & interés & $4,88 \%$ & $100 \%$ \\
$(18,81 \%)$ & Enseñanza programada & $0 \%$ & \\
& Programa individual & $0 \%$ & $100 \%$ \\
\hline Creativos (7,34\%) & Enseñanza modulada & $100 \%$ & \\
\hline & Sinéctica & $50 \%$ & $100 \%$ \\
Participativos y socializadores (3,67\%) & Enseñanza recíproca & $25 \%$ & \\
& Grupos reducidos & $25 \%$ & \\
\hline Cognoscitivos $(0,46 \%)$ & Microenseñanza & $0 \%$ & $100 \%$ \\
\hline
\end{tabular}

Fuente: Elaboración propia

\section{DISCUSIÓN Y CONCLUSIONES}

El primer objetivo de estudio buscaba conocer las creencias de los participantes sobre las metodologías activas y participativas de la enseñanza por competencias. Los resultados del grupo de discusión y las entrevistas encontraron que el profesorado era consciente de la necesidad de renovar la práctica hacia estas metodologías. Además, las ideas de los participantes fueron congruentes en el análisis de contenido, el grupo de discusión y las entrevistas. En este sentido, los profesores afirmaron conocer estrategias para acercarse al enfoque por competencias, como el uso de aprendizaje cooperativo, una orientación compleja, interdisciplinar y contextualizada de los aprendizajes o la cesión de responsabilidad y autonomía al alumnado. Estos resultados coinciden con los obtenidos por Hortigüela et al. (2015a, 2015b) y Méndez-Giménez et al. (2013), quienes encontraron que los profesores identifican el trabajo competencial con el desarrollo de metodologías cooperativas y participativas. Este hecho puede ser un adecuado punto de partida para alcanzar un cambio metodológico en las aulas de los participantes. De hecho, como encontró Hong (2012), uno de los principales cambios que se produjeron para aplicar metodologías centradas en el alumno fue que el profesorado asumió la necesidad renovar la metodología y utilizar estos estilos de enseñanza.

En esta línea, otro aspecto relevante de los hallazgos del estudio es la visión positiva de la forma de trabajar en la EF y la formación de sus recursos humanos para renovar la 
metodología. Se corroboran los hallazgos de Amin y Rajaei (2011), Fernández-Rivas y Espada (2016), Hortigüela et al. (2016), Méndez-Alonso et al. (2015) y Méndez-Giménez et al. (2013), que encontraron cómo los profesores de EF manifiestan una mayor predisposición hacia estas metodologías. Por tanto, este parece otro punto de apoyo para lograr una renovación metodológica en los contextos investigados. En contraposición con estos apoyos, los resultados del grupo de discusión y las entrevistas evidenciaron que, si bien los participantes consideran posible una combinación de metodología tradicional y otra más activa, los profesores no apuntaron a una utilización frecuente de estos métodos.

Partiendo de estas ideas de los participantes, se analiza el segundo objetivo de este estudio: "analizar la metodología desarrollada en el quehacer diario de los profesores de EF". Los hallazgos contrastan con las opiniones de los participantes en el grupo de discusión y entrevistas. Al menos en las sesiones observadas, se puso de manifiesto el predominio de estilos de enseñanza tradicionales, mientras que aquellos más activos, como los participativos, socializadores e individualizadores, ocuparon un papel menos relevante. Estos resultados coinciden con los encontrados en otras investigaciones (AKTOP; KARAHAN, 2012; ARAUJO; CAMPOS, 2006; COTHRAN et al., 2005; FERREIRA; PEREIRA, 2009; HEIN et al., 2012; HODGES; COTHRAN, 2003; VARGAS; SILVA, 2008). Deacuerdo con estos estudios, parece que las prácticas profesionales de los profesores de EF se alejan de planteamientos metodológicos activos, participativos y centrados en el alumno. Al respecto, la EF en los entornos investigados parece más cercana a planteamientos tradicionales desarrollados desde premisas directivas y técnicas, donde el profesorado se erige como el actor principal en el proceso de enseñanza. La interpretación de estos resultados lleva a concluir que la renovación metodológica, en el marco de políticas competenciales, no llega a la práctica en los contextos investigados, lo que coincide con la baja materialización de este enfoque (HORTIGÜELA et al., 2015a;MÉNDEZ-ALONSO et al., 2015; MONARCA; RAPPOPORT, 2013).Parece relevante la baja consolidación de estas metodologías, más aún cuando se ha advertido de las potencialidades de las metodologías activas en el aprendizaje del alumnado (CORINA et al., 2011; LAAL et al., 2014) y de la amplia conceptualización e indagación que se ha producido sobre la metodología en EF. Asimismo, en el caso de España, la presión reguladora de la normativa no parece estar consiguiendo aprovechar los beneficios que pueden generar el uso de estas metodologías, como la mayor predisposición y rendimiento de los alumnos (ÁLVAREZ-ARREGUI et al., 2013; AMIN; RAJAEI, 2011; CORINA et al., 2011).

Las ideas sobre los dos primeros objetivos de estudio permiten analizar el tercer objetivo de estudio, "conocer el grado de relación entre las creencias sobre estas metodologías y su desarrollo en las aulas de los participantes". No se obtuvo una linealidad entre las creencias de los participantes y su desempeño en el aula. A pesar de que los participantes conocen estas metodologías y manifiestan una cierta predisposición para su desarrollo, su práctica habitual se identificó predominantemente con un enfoque tradicional de la EF. Diversos estudios han indagado sobre los motivos de esta baja materialización de las metodologías activas y participativas y sus hallazgos apuntan a la falta de formación y la desinformación como una de las principales barreras para que se supere la lógica técnica tradicional e imperante en la EF (BARRACHINA; BLASCO, 2012; FERREIRA; PEREIRA, 2009; RAMÍREZ, 2015;VARGAS; SILVA, 2008; ZAPATERO-AYUSO et al., 2012). No obstante, Hong (2012), Hortigüela et al. (2015a, 2015b) y Méndez-Giménez et al. (2013) aseveran que el cambio metodológico no parece una responsabilidad única de los profesores. Para alcanzarlo, los implicados en 
políticas educativas, las instituciones dedicadas a la preparación del profesorado y los centros educativos deben impulsar esta renovación con recursos, formación y favoreciendo el trabajo en equipo en la escuela (HONG, 2012; MONARCA; RAPPOPORT, 2013; VARGAS;SILVA, 2008).

Una limitación del estudio es la participación de un grupo reducido de profesores. En consecuencia, en futuras investigaciones sería necesario ampliar la muestra de los participantes (profesorado, equipos directivos, responsables de las administraciones educativas e instituciones de formación del profesorado) con la finalidad de profundizar en los resultados del problema objeto de estudio. No obstante, el estudio pretende valorar una realidad concreta, abordando la complejidad del problema en un contexto y permitiendo profundizar sobre las creencias, actitudes y el desempeño práctico de los participantes sobre la metodología de enseñanza. Este análisis abre un punto relevante de reflexión y debate, al menos en los contextos de los participantes, sobre los motivos por los cuáles existe un distanciamiento entre las creencias y actitudes positivas del profesorado de EF hacia estas metodologías activas y su reducido desarrollado en sus prácticas profesionales.

\section{REFERENCIAS}

AKTOP, Abdurrahman; KARAHAN, Nilüfer.Physical Education Teacher's Views of Effective Teaching Methods in Physical Education.Procedia-Social and BehavioralSciences, v.46, p.19101913, 2012.Disponible en: <https://www.sciencedirect.com/science/article/pii/S1877042812015303>. Acceso en: 19 mar. 2018.

ÁLVAREZ-ARREGUI, Emilio; RODRÍGUEZ-MARTÍN, Alejandro; SAN FABIÁN, José Luis. Metodologías y recursos didácticos en los títulos de grado. ¿Qué hace el profesorado y qué quiere el alumnado? Tendencias pedagógicas, Madrid, n.22, 2013.Disponible en: <https:// repositorio.uam.es/bitstream/handle/10486/14082/66026 7.pdf? sequence=1\&isAllowed=y>. Acceso en: 19 mar. 2018.

ALVIRA, Francisco. Metodología de la evaluación de programas. Madrid: CIS, 1991.

ARAUJO, Mercedes; CAMPOS, Moraima. La praxis pedagógica en la educación física y los estilos de enseñanza predominantes en los docentes de educación básica. Educare, v.10, n.3, p.165-191, 2006. Disponible en: <http://revistas.upel.edu.ve/index.php/educare/article/ view/144/124>. Acceso en: 19 mar. 2018.

AMIN, Maghsood; RAJAEI, Malihe. The comparison of students and educators preferred teaching styles in teacher educating centres. Procedia-Social and BehavioralSciences, v.15, p.1875-1880, 2011. Disponible en: <https://www.sciencedirect.com/science/article/pii/ S1877042811005659>. Acceso en: 24 mar. 2018.

ARNAU, Jaime; ANGUERA, María-Teresa; GÓMEZ, Jaime. Metodología de la investigación en ciencias del comportamiento. Murcia: Universidad de Murcia, 1990.

BARDIN, Laurence. El análisis de contenido. Madrid: Akal, 1986.

BARRACHINA, Julio; BLASCO, Josefa. Análisis del desarrollo de las competencias básicas en el currículum de la Educación Física en la ESO en la Marina Baixa. Un estudio de caso. Apunts: 
Educación física y deportes, n.110, p.36-44, 2012. Disponible en: <http://www.revista-apunts.com/es/ hemeroteca?article=1563>. Acceso en: 19 mar. 2018.

BISQUERRA, Rafael. Métodos de investigación educativa. Barcelona: Ceac, 1989.

CALLEJO, Javier. El grupo de discusión: introducción a una práctica de investigación. Barcelona: Ariel, 2001.

COLÁS, María-Paz; BUENDÍA, Leonor. Investigación educativa. Sevilla: Alfar, 1992.

CONFEDERAÇÃO NACIONAL DOS TRABALHADORES EM EDUCAÇÃO. Parte I. Ajustes estructurales y educación en Brasil. En Consejo Latinoamericano de Ciencias Sociales (CLCS). Las reformas educativas en los países del Cono Sur: un balance crítico. Buenos Aires: CLCS, 2005. p.173-188. Disponible en: <http://biblioteca.clacso.edu.ar/clacso/coediciones/20100823015222/10BrP1C1.pdf $>$. Acceso en: 23 mar. 2018.

COSTA, Vera. Prática de Educação Física: Modelo de reprodução ou perspectiva de transformação. São Paulo, IBRASA, 1987.

COTHRAN, Donetta et al. A cross-cultural investigation of the use of teaching styles. Research Quarterly for Exercise and Sport, v.76, n.2, p.193-201, 2005.Disponible en: <http://citeseerx.ist.psu.edu/viewdoc/ download?doi=10.1.1.565.8377\&rep=rep1\&type=pdf>. Acceso en: 19 mar. 2018.

CORINA, lureaa et al.. The Study of the Relation between the Teaching Methods and the Learning Styles - The Impact upon the Students' Academic Conduct. Procedia-Social and Behavioral Sciences, v.11, p.256-260. Disponible en: <https://www.sciencedirect.com/science/article/pii/S1877042811000747>. Acceso en: 24 mar. 2018.

ESPAÑA. Ley Orgánica 2/2006, de 3 de mayo, de Educación. Boletín Oficial del Estado de 4 de Mayo de 2006. Disponible en: <https://www.boe.es/buscar/pdf/2006/BOE-A-2006-7899-consolidado.pdf>. Acceso en: 05 mayo 2018.

ESPAÑA. Ley Orgánica 8/2013, de 9 de diciembre, para la mejora de la calidad educativa. Boletín Oficial del Estado de 10 de Diciembre de 2013. Disponible en: <https://www.boe.es/buscar/pdf/2013/ BOE-A-2013-12886-consolidado.pdf>. Acceso en: 05 mayo 2018.

FERNÁNDEZ-RIVAS, María; ESPADA, María. Actitud del profesorado de Educación Física frente al aprendizaje cooperativo. Movimento, v.22, n.2, p.861-876, 2016.Disponible en: <http://seer.ufrgs.br/ index.php/Movimento/article/view/61620/38856>. Acceso en: 19 mar. 2018.

FERREIRA, Nilton; PEREIRA, Sheila-Aparecida. Conhecimento e Aplicação de Métodos de Ensino para os Jogos Esportivos Coletivos na Formação Profissional em Educação Física. Movimento,v.15, n.1, p.117-144, 2009. Disponible en: <http://www.seer.ufrgs.br/index.php/Movimento/article/view/2086/4829>. Acceso en: 24 mar. 2018.

FIGUERAS, Sara et al. Competencias básicas y educación física: estudios e investigaciones. Apunts. Educación Física y deportes, n.123,p.34-43, 1.er trim.2016. Disponible en: <http://www.raco.cat/index. php/ApuntsEFD/article/view/306849>. Acceso en: 23 mar. 2018.

GIL-FLORES, Javier. La metodología de investigación mediante grupos de discusión. Enseñanza. Enseñanza \&Teaching. Revista interuniversitaria de Didáctica, v.10, n.11, p.199-212, 1993.Disponible en: <http://revistas.usal.es/index.php/0212-5374/article/view/4179>. Acceso en: 20 mar. 2018.

GLASER, Barney; STRAUSS, Anselm.The discovery of Grounded Theory: Strategies for qualitative research.New York: Aldine de Gruyter, 1967. 
GOLDBERGER, Michael; ASHWORTH, Sara; BYRA, Mark. Spectrum of Teaching Styles Retrospective 2012, Quest, n.64, v.4, p.268-282, 2012.Disponible en: <https://www.tandfonline. com/doi/abs/10.1080/00336297.2012.706883>. Acceso en: 20 mar. 2018.

GUTIÉRREZ, Jesús. Dinámica del grupo de discusión. Madrid: CIS, 2008.

HEIN, Vello etal. The relationship between teaching styles and motivation to teach among physical education teachers.Journal of Sports Science \&Medicine, v.11, n.1, p.123-130, 2012. Disponible en: <https://www.jssm.org/vol11/n1/18/v11n1-18text.php>. Acceso en: 20 mar. 2018.

HODGES, Pamela; COTHRAN, Donetta. Physical education teachers' self reported use and perceptions of various teaching styles. Learning and Instruction, n.13, p.597-609, 2003. Disponible en: <https://www.sciencedirect.com/science/article/pii/S1877042812015303>. Acceso en: 20 mar. 2018.

HONG, Won-Pyo.An international study of the changing nature and role of school curricula: from transmitting content knowledge to developing students' key competencies. Asia Pacific Education Review, v.13,n.1, p.27-37, 2012.

HORTIGÜELA, David; ABELLA, Víctor; PÉREZ-PUEYO, Ángel. Percepción de equipos directivos y docentes de Educación Física sobre el proceso de implantación y desarrollo de las competencias básicas en la ciudad de Burgos. Cultura, Ciencia y Deporte, v.10, n.28, p.19-30, 2015a. Disponible en: <http://www.redalyc.org/pdf/1630/163036903006.pdf>. Acceso en: 20 mar. 2018.

HORTIGÜELA, David; ABELLA, Víctor; PÉREZ-PUEYO, Ángel. Percepciones de directivos y profesorado de Educación Física sobre las Competencias Básicas. Enseñanza \&Teaching, v.33, n.1, p.83-103, 2015b. Disponible en: <http://revistas.usal.es/index.php/0212-5374/article/viewFile/ et201533183103/13308>. Acceso en: 20 mar. 2018.

IBÁÑEZ, Jesús. Más allá de lasociología: el grupo de discusión: teoría y práctica. 5.ed. Madrid: Siglo XXI, 2003.

ION, Georgeta; CANO, Elena. La formación del profesorado universitario para la implementación de la evaluación por competencias. Educación XX1, v.15, n.2, p.249-270, 2012.Disponible en: $<$ <http://revistas.uned.es/index.php/educacionXX1/article/view/141/104>. Acceso en: 20 mar. 2018.

JALLADE, Jean-Pierre. International Approaches to Education: a review of some major cooperative programmes. European Journal of Education, v.46, n.1, p.7-24, 2011. Disponible en: $<$ https://onlinelibrary.wiley.com/doi/abs/10.1111/j.1465-3435.2010.01469.x>. Acceso en: 23 de mar. 2018.

KLEIBRINK, Alexander. The EU as a Norm Entrepreneur: the case of lifelong learning. European Journal of Education, v.46, n.1, p.70-84, 2011. Disponible en: <https://onlinelibrary.wiley.com/doi/fu I/10.1111/j.1465-3435.2010.01461.X>. Acceso en: 23 de mar. 2018.

KRUEGER, Richard. El grupo de discusión. Guía práctica para la investigación aplicada. Madrid: Pirámide, 1991.

LAAL, Marjan; KHATTAMI-KERMANSHAHI, Zhina; LAAL, Mozhgan.Teaching and Education; Collaborative Style.Procedia-Social and BehavioralSciences, v.116, p.4057-4061, 2014. Disponible en: <https://www.sciencedirect.com/science/article/pii/S1877042814009070>. Acceso en: 24 mar. 2018. 
LUKAS, José; SANTIAGO, Karlos. Evaluación educativa. Madrid: Alianza, 2004.

MARTINS, Idalina et al. Las competencias en las políticas de currículum de ciencias: los casos de Brasil y Portugal. Revista mexicana de investigación educativa, v.18, n.56, p.37-62, 2013. Disponible en: <http://www.scielo.org.mx/pdf/rmie/v18n56/v18n56a3.pdf>. Acceso en: 29 mar.2018.

MARTÍNEZ-MEDIANO, Catalina. Técnicas e instrumentos de recogida y análisis de datos. Madrid: UNED, 2004.

MARTÍNEZ-SALGADO, Carolina. El muestreo en investigación cualitativa. Principios básicos y algunas controversias. Ciência \&Saúde Coletiva, v.17, n.3, p.613-619, 2012. Disponible en: <http://www.scielo.br/pdf/csc/v17n3/v17n3a06.pdf>. Acceso en: 23 mar. 2018.

MÉNDEZ-ALONSO, David; MÉNDEZ-GIMÉNEZ, Antonio; FERNÁNDEZ-RíO, Francisco-Javier. Análisis y valoración del proceso de incorporación de las Competencias Básicas en Educación Primaria. Revista de Investigación Educativa, v.33, n.1, p.233-246, 2015.Disponible en: <http:// revistas.um.es/rie/article/view/183841>. Acceso en: 20 mar. 2018.

MÉNDEZ-GIMÉNEZ, Antonio; SIERRA, Beatriz; MAÑANA, Jorge. Percepciones y creencias de los docentes de Primaria del Principado de Asturias sobre las competencias básicas. Revista de Educación, n.362, p.737-761, 2013. Disponible en: <https://recyt.fecyt.es/index.php/Redu/article/ view/16544>. Acceso en: 20 mar. 2018.

MENDONÇA, Marcílio-Barbosa; SOARES, Marcelo; ELIETE, Maria. A análise de conteúdo como forma de tratamento dos dados numa pesquisa qualitativa em Educação Física escolar. Movimento, v.16, n.3, p.31-49, 2010.Disponible en: <http://seer.ufrgs.br/index.php/Movimento/ article/view/11546>. Acceso en: 20 mar. 2018.

MONARCA, Héctor; RAPPOPORT, Soledad. Investigación sobre los procesos de cambio educativo: El caso de las competencias básicas en España.Revista de Educación, n. esp., p.54-78, 2013.Disponible en: <https://www.mecd.gob.es/dctm/revista-de-educacion/articulosre2013/ re201303.pdf?documentld=0901e72b8176d627>. Acceso en: 20 mar. 2018.

MOSSTON, Muska; ANSWORTH, Sara. The spectrum of Teaching styles: from command to discovery. New York: Longsman, 1990.

OLIVEIRA, Amauri. Metodologias emergentes no ensino da educação física. Revista da Educação Física/UEM, n.8, v.1, p.21-27,1997.Disponible en: <http://eduem.uem.br/ojs/index.php/ RevEducFis/article/view/3868>. Acceso en: 20 mar. 2018.

PATTON, Michael-Quinn. How to use qualitative methods in evaluation. BeverlyHills: Sage, 1987.

PÉREZ-SERRANO, Gloria. Investigación cualitativa. Retos e interrogantes. II. Técnicas y análisis de datos. Madrid: La Muralla, 1998.

RAMÍREZ, Antonio. La formación del profesorado de Educación Primaria ante las competencias básicas. Revista Electrónica Interuniversitaria de Formación del Profesorado, v.18, n.3, p.199214, 2015. Disponible en: <http://revistas.um.es/reifop/article/view/193811>. Acceso en: 20 mar. 2018.

RUIZ-OLABUÉNAGA, José Ignacio. Metodología de la investigación cualitativa. 2.ed.. Bilbao: Universidad de Deusto, 1999. 
SICILIA-CAMACHO, Álvaro. La investigación de los estilos de enseñanza en la Educación Física: un viejo tema para un nuevo siglo. Barcelona: Wanceulen, 2001.

SICILIA-CAMACHO, Álvaro; DELGADO-NOGUERA, Miguel-Ángel. Educación Física y estilos de enseñanza. Barcelona: INDE, 2002.

SOARES, Carmen Lúcia et al..Metodologia do ensino de educação física. Campina: Cortez, 1992.

STRAUSS, Anselm; CORBIN, Juliet. Basics of qualitative research: Techniques and procedures for developing Grounded Theory. 2.ed. California: SAGE, 1998.

TEJADA, José. La alternancia de contextos para la adquisición de competencias profesionales en escenarios complementarios de educación superior: marco y estrategia. Educación XX1, v.15, n.2, p.19-40, 2012.Disponible en: <http://revistas.uned.es/index.php/educacionXX1/article/ view/125>. Acceso en: 20 mar. 2018.

VALLES, Miguel. Entrevistas cualitativas. Madrid: CIS, 2002.

VARGAS, Daniele; SILVA, Maristela da. Analysis of theoretical-methodological knowledge of CEFD/UFSM physical education.Professors in relation to their pedagogical practice. Movimento,v.14, n.2, p.63-82.Disponible en: <www.seer.ufrgs.br/Movimento/article/ download/2315/3359>. Acceso en: 24 mar. 2018.

ZAPATERO-AYUSO, Jorge-Agustín, GONZÁLEZ-RIVERA, María-Dolores; CAMPOSIZQUIERDO, Antonio. La formación de los docentes de Educación Física en torno a la enseñanza por competencias a través de un grupo de discusión. Emásf, Revista Digital de Educación Física, v.3, n.17, p.1-15, 2012.Disponible en:<http://emasf.webcindario.com/ Contribucion del area de EF al desarrollo de las competencias .pdf>. Acceso en: 20 mar. 2018. 
